\title{
ROL DEL DISTRÉS PSICOLÓGICO EN LA RELACIÓN ENTRE PERCEPCIÓN DE ENFERMEDAD Y CALIDAD DE VIDA EN PACIENTES CON CÁNCER DE MAMA
}

\author{
THE ROLE OF PSYCHOLOGICAL DISTRESS IN THE RELATIONSHIP BETWEEN ILLNESS \\ PERCEPTION AND QUALITY OF LIFE IN PATIENTS WITH BREAST CANCER
}

Daniela Gercovich, Pablo Luis López, Diego Bortolato, Patricia Margiolakis, Mirta Morgenfeld, Laura Rosell, Ernesto Gil Deza

Instituto William Osler, Paraguay 2935 (C1425BRI), Ciudad Autónoma de Buenos Aires, Argentina

Resumen

Objetivo: estudiar las asociaciones entre las dimensiones de percepción de enfermedad y la calidad de vida, evaluando el rol modulador del distrés psicológico en pacientes con cáncer de mama e identificando cuáles de dichas dimensiones explicaba en mayor medida la variabilidad en los distintos aspectos de la calidad de vida.

Método: setenta y cinco pacientes fueron evaluadas con el Cuestionario de Percepción de Enfermedad Breve y el Cuestionario de Calidad de Vida de la Organización Europea para la Investigación y el Tratamiento de Cáncer (EORTC QLQC30). Se calcularon correlaciones bivariadas y parciales para evaluar las asociaciones entre las dimensiones de percepción de enfermedad y distintos aspectos de la calidad de vida, controlando por una medida de distrés. Posteriormente, se ha realizado un análisis de regresión lineal para evaluar las dimensiones de percepción de enfermedad que podrían explicar la variabilidad en la calidad de vida.

Resultados: si bien se encontraron asociaciones significativas entre las subescalas de percepción de enfermedad y calidad de vida, la mayoría de ellas perdían su significación cuando se controlaba por distrés. En los modelos

\section{Abstract}

Objective: To evaluate the associations between the illness perception dimensions and quality of life, assessing the modulatory role of psychological distress in patients with breast cancer, identifying which of these dimensions explained further variability in the different aspects of the quality of life.

Methods: Seventy-five patients were evaluated with the Brief Illness Perception Questionnaire and the European Organization for Research and Treatment of Cancer (EORTC) Quality of Life Questionnaire (QLQ-30). We calculated bivariate and partial correlations to evaluate the associations between the illness perception dimensions and different aspects of quality of life, controlling for a distress measure. Subsequently, we performed linear regression analysis to evaluate the illness perception dimensions that could explain the variability in the quality of life scores.

Results: Although significant associations between subscales of perception of illness and quality of life were found, most of them lost their significance when controlled by distress. In the regression models, variables that best predicted the variability in the quality of life were psychopathological diagnostic and distress.

\section{Correspondencia:}

Instituto William Osler, Paraguay 2935 (C1425BRI),

Ciudad Autónoma de Buenos Aires,

Argentina,

E-mail: dg@williamosler.com.ar 
de regresión, las variables que mejor predecían la variabilidad de la calidad de vida fueron el diagnóstico psicopatológico y el distrés.

Conclusiones: de acuerdo a los resultados del presente estudio, el distrés psicológico y el diagnóstico psicopatológico fueron las dos variables que más explicaban la variabilidad de la calidad de vida. Por tal motivo se hace imprescindible profundizar en el rol de dichas variables en la calidad de vida y en la morbimortalidad asociada a ellas.

Palabras clave: Percepción de Enfermedad, calidad de vida, distrés, psicooncología.
Conclusions: According with the study results, psychological distress and psychopathological diagnostic were the two variables that explained better the variability in the quality of life. For this reason it is essential to learn more about the role of these variables on the quality of life and morbidity and mortality associated with them.

Keywords: Illness Perception, quality of life, distress, psychooncology.

cepción de enfermedad más estudiadas se encuentran las variables que identifican la presencia o ausencia de una enfermedad, incluyendo el nombre de la misma y a los signos o síntomas (identidad), las creencias sobre la etiología (causas), sobre los efectos y las implicaciones físicas, emocionales, sociales y económicas de la enfermedad (consecuencias), sobre la duración y evolución de la enfermedad (tiempo), y las creencias sobre cómo puede controlarse la enfermedad o recuperarse de la misma (control/cura) ${ }^{(3-6)}$.

Existen investigaciones previas sobre percepción de enfermedad en pacientes con distintas patologías, que apoyan empíricamente la hipótesis de que la teoría que los pacientes tienen sobre la enfermedad es crítica para la adaptación exitosa a la enfermedad médica ${ }^{(7,8)}$. Particularmente en el campo de la enfermedad oncológica, existe evidencia empírica en estudios realizados con mujeres con cáncer ginecológico y de mama ${ }^{(9-11)}$ y cáncer de pulmón ${ }^{(12)}$.

Previo a este estudio, se ha realizado una investigación en pacientes con cáncer de cabeza y cuello ${ }^{(13)}$ que evaluó en qué medida la percepción de enfermedad de los pacientes determinaba su calidad de vida. Tomando como referencia a dicho estudio, decidimos evaluar la relación 
entre ambos constructos en una muestra de pacientes con cáncer de mama con el objetivo de identificar las dimensiones de percepción de enfermedad que se asocian con distintos aspectos de la calidad de vida. A diferencia del estudio citado, en este trabajo hemos decidido incluir una medida de distrés psicológico para evaluar su rol en la asociación de las variables mencionadas. Esta inclusión la consideramos imprescindible dado que ha sido ampliamente reportada la presencia de distrés en este tipo de pacientes así como también el modo en que dicho distrés se relaciona con la evolución de la enfermedad y los indicadores de morbi-mortalidad ${ }^{(14)}$. Sumado a lo anterior, existe evidencia que sostiene que los síntomas de ansiedad y depresión se asocian a limitaciones funcionales en los pacientes con cáncer ${ }^{(15)}$. Un trabajo publicado recientemente ${ }^{(16)}$ fue el primero en considerar una medida vinculada con el distrés como factor modulador de la asociación entre percepción de enfermedad y calidad de vida.

\section{MÉTODO}

\section{Diseño}

El presente es un estudio ex post facto retrospectivo ${ }^{(17)}$, en el cual se definen como variables independientes a las dimensiones de percepción de enfermedad y como variables dependientes a la calidad de vida de los pacientes.

\section{Participantes}

Se evaluaron 75 pacientes mujeres que ingresaron de manera consecutiva en el servicio de salud mental de un instituto especializado en enfermedad oncológica. Las pacientes fueron evaluadas en la entrevista de admisión del mencionado servicio. Todos los participantes han sido informados de las características y objetivos del estudio, prestando su consentimiento para su inclusión en el mismo.

\section{Instrumentos}

Cuestionario Breve de Percepción de Enfermedad (BIPQ): es un cuestionario autoadministrable de nueve ítems diseñado por Broadbent et al. ${ }^{(18)}$. Este instrumento es una medida de las representaciones cognitivas y emocionales de los pacientes con respecto a su enfermedad. Está compuesto por las siguientes dimensiones: identidad, consecuencias, duración, control sobre la enfermedad, control del tratamiento, preocupación, comprensión y respuesta emocional. Además indaga sobre las causas que los pacientes atribuyen a su enfermedad. La interpretación de este cuestionario es sencilla: un aumento en el puntaje del ítem indica un incremento lineal de la dimensión que mide. La validación lingüística al español fue desarrollada por Pacheco-Huergo y colaboradores ${ }^{(19)}$. En el estudio psicométrico realizado posteriormente por la misma autora se registró un índice de consistencia interna del $0,67^{(20)}$. Con respecto a la validez estructural, la autora propone un análisis factorial confirmatorio, en donde los datos se estructuraron en dos componentes. Un componente relacionado con la representación emocional frente a la amenaza de la enfermedad y agrupaba a los ítems identidad, consecuencias, respuesta emocional y preocupación. El segundo componente estaba relacionado con la representación cognitiva y el afrontamiento y agrupó los ítems control del tratamiento, control personal y comprensión de la enfermedad. El instrumento también presentó buenos indicadores de validez externa y discriminativa.

Cuestionario de Calidad de Vida para Cáncer de la European Organization for Research and Treatment of Cancer (EORTC-QLQ-30)(21): es un instrumento para evaluar de manera específica la ca- 
lidad de vida en sus diferentes dimensiones en pacientes con cáncer. El mismo consta de 30 ítems distribuidos en cinco escalas funcionales (funcionamiento físico; rol; funcionamiento social; funcionamiento emocional y funcionamiento $\mathrm{cog}$ nitivo), y tres escalas de síntomas (fatiga, dolor, náuseas y vómitos). Asimismo, el cuestionario incorpora una escala global de salud/calidad de vida y algunos ítems individuales que evalúan distintos síntomas de la enfermedad y/o del tratamiento (disnea, insomnio, pérdida de apetito, estreñimiento, diarrea e impacto financiero). Las preguntas se refieren a un período de tiempo de una semana y se emplea un formato de respuesta tipo Likert. Para el análisis en este estudio se utilizaron las 6 dimensiones principales (esto es, la escala global y las cinco de funcionamiento) y la subescala de dolor. Se ha utilizado la versión española de Arrarás et al. ${ }^{(22)}$.

Escala de Ansiedad y Depresión Hospitalaria $(H A D S)^{(23)}$ : es un instrumento especialmente diseñado para identificar y medir el malestar psicológico en pacientes con patología médica no psiquiátrica. El mismo apunta a relevar las dos formas más comunes de perturbación psicológica en pacientes con patología médica, esto es, la ansiedad y la depresión. Se trata de un instrumento breve (14 ítems) de fácil administración, que en promedio toma entre 2 y 6 minutos para su aplicación y que ha mostrado ser altamente aceptable para los pacientes. En su construcción se descartaron síntomas y signos somáticos de la ansiedad y la depresión que pueden confundirse fácilmente con las alteraciones primarias propias de los cuadros médicos de base, problema que sí presentan otros instrumentos de evaluación psicológica. Asimismo se privilegiaron los ítems que reflejan perturbaciones emocionales leves y moderadas, más adecuadas a la presentación de los pacien- tes no psiquiátricos. Por estos motivos, se trata de un instrumento de extenso uso en la evaluación de los concomitantes psicológicos de variadas patologías médicas, traducido y validado en diferentes idiomas y países. Al presente existen dos importantes revisiones de sus propiedades psicométricas y de sus aplicaciones en investigación ${ }^{(24,25)}$. La primera revisión resalta un comportamiento adecuado de esta herramienta para evaluar cambios en el estado psicológico producidos por las intervenciones psicosociales (sensibilidad al cambio). Esta escala ha sido ampliamente utilizada en pacientes oncológicos para medir distrés ${ }^{(26)}$. En este estudio se ha utilizado la versión adaptada en la Ciudad de Buenos Aires para muestras de pacientes oncológicos ${ }^{(27)}$.

\section{Análisis de datos}

Las asociaciones entre las dimensiones de percepción de enfermedad y las distintas subescalas de funcionamiento de calidad de vida se calcularon utilizando el coeficiente de correlación de Pearson. Luego se realizaron correlaciones parciales entre las mismas variables controlando por los puntajes de la HADS.

Finalmente, a fin de evaluar el valor predictivo de las dimensiones de percepción de enfermedad, se realizó un análisis de regresión lineal por pasos. Las variables Edad y Estadio de la Enfermedad se ingresaron en el paso 1, luego se introdujeron en el paso 2 los diagnósticos psicopatológicos y el puntaje total de la HADS en el modelo con el fin de controlar sus posibles efectos confundidores en las asociaciones. Finalmente, para limitar el número de variables, solamente se decidió incluir aquellas subescalas de la BIPQ que mostraran un alto grado de correlación parcial con las subescalas de la QLQ-30 controlando por los puntajes de la HADS. 
Los análisis estadísticos fueron realizados a través del paquete estadístico SPSS 17.0 .

\section{Resultados}

La media de edad de la muestra fue de 55,96 (DS=12,02). Con respecto al estadio de la enfermedad, 17 mujeres presentaban estadio 1, 35 estadio 2, 10 estadio 3 y 10 estadio 4 (3 casos perdidos). En cuanto al tratamiento recibido, el $4,1 \%$ de la muestra recibía tratamiento paliativo $A$, el 4,1 $\%$ tratamiento Paliativo B, el 33,8 \% tratamiento adyuvante y el $8,1 \%$ se encontraba en tratamiento control.

Con respecto al diagnóstico psicopatológico, 37 pacientes estaban diagnostica- das con trastorno adaptativo y 6 pacientes cumplían criterios para trastorno depresivo mayor. El resto de las pacientes no cumplía criterios para ningún trastorno mental, de acuerdo al Manual Diagnóstico y Estadístico de los Trastornos Mentales (DSM IV-TR) ${ }^{(28)}$.

Los estadísticos descriptivos de las subescalas de los instrumentos utilizados se detallan en la tabla 1.

Las correlaciones entre las dimensiones de percepción de enfermedad y los distintos aspectos de la calidad de vida se resumen en la tabla 2 .

Como puede observarse la subescala de Identidad es la que más se asoció a las distintas dimensiones de calidad de vida, correlacionando de manera inversa con casi todas ellas, a excepción de QLQ-30:

Tabla 1. Estadísticos descriptivos de las subescalas de la BIPQ y del QLQ-30.

\begin{tabular}{|llll|}
\hline \multicolumn{1}{c}{ Subescala } & & \multicolumn{2}{c|}{$95 \%$ IC } \\
\cline { 4 - 4 } BIPQ & Media (DS) & LI & LS \\
Consecuencias & $6,54(2,85)$ & 5,88 & 7,20 \\
Duración & $5,34(3,39)$ & 4,55 & 6,12 \\
Control sobre la enfermedad & $5,36(3,21)$ & 4,62 & 6,11 \\
Control sobre el tratamiento & $8,18(2,73)$ & 7,54 & 8,81 \\
Identidad & $4,32(3,21)$ & 3,58 & 5,07 \\
Preocupación & $6,93(3,29)$ & 6,18 & 7,68 \\
Comprensión & $6,82(3,22)$ & 6,08 & 7,57 \\
Respuesta Emocional & $7,20(2,96)$ & 6,52 & 7,89 \\
& & & \\
QLQ-30 & & & 60,89 \\
Calidad de vida global & $55,52(23,17)$ & 50,15 & 78,57 \\
Funcionamiento Físico & $73,06(23,76)$ & 67,56 & 70,58 \\
Funcionamiento de Rol & $63,96(28,54)$ & 57,35 & 51,33 \\
Funcionamiento Emocional & $45,01(27,31)$ & 38,68 & 78,47 \\
Funcionamiento Cognitivo & $72,07(27,61)$ & 65,68 & 66,72 \\
Funcionamiento Social & $59,23(32,31)$ & 51,75 & \\
\hline
\end{tabular}

Nota: IC = intervalo de confianza; $\mathrm{LI}=$ límite inferior; $\mathrm{LS}$ = límite superior. 


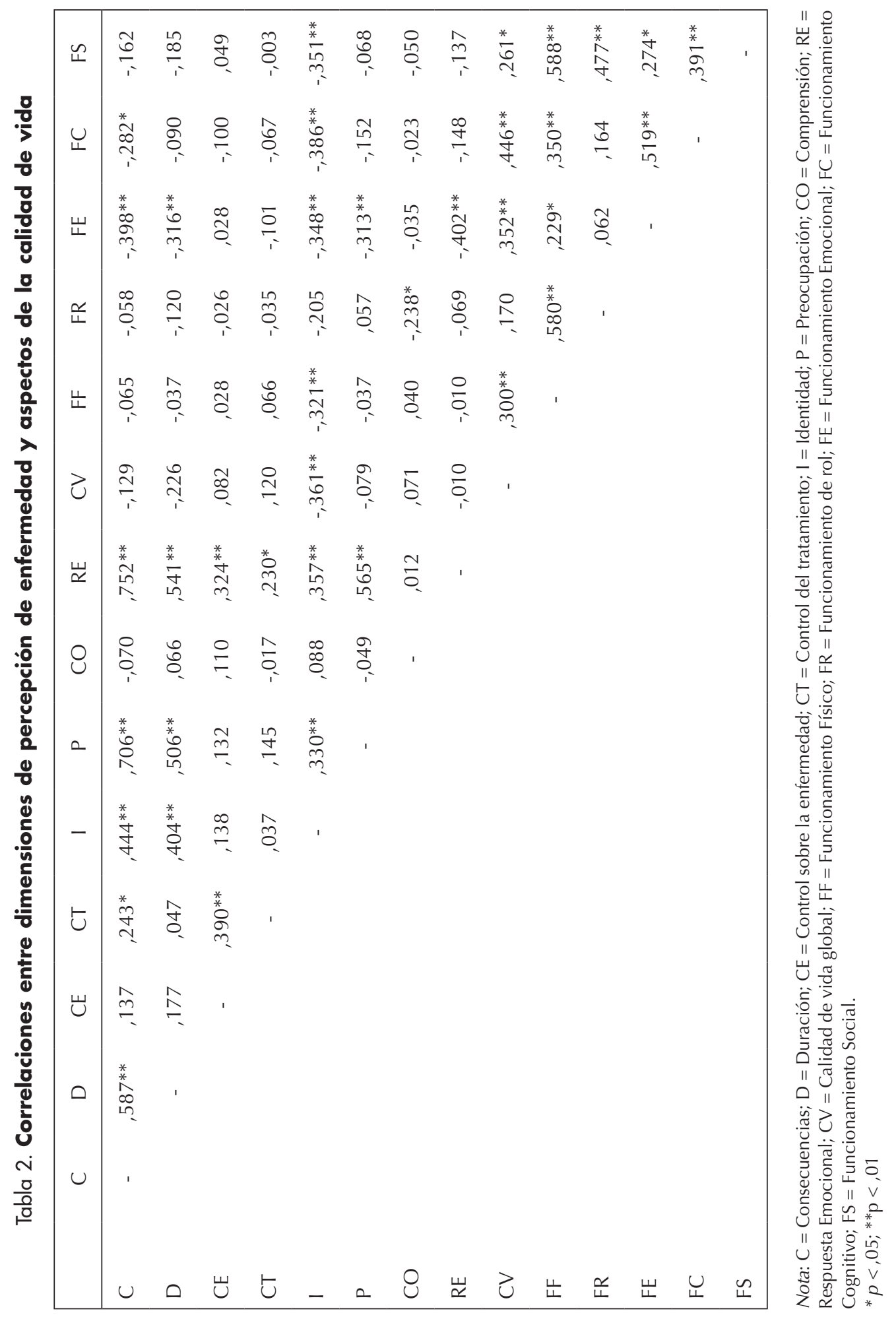


Funcionamiento de Rol. En tanto, la subescala de Consecuencias se asoció a un peor Funcionamiento Emocional y Cognitivo. Una asociación similar se encuentra entre la subescala de Preocupación y Funcionamiento Emocional y entre Comprensión y Funcionamiento de Rol. Finalmente la subescala de Respuesta emocional se asoció de manera inversa con la dimensión de Funcionamiento Emocional.

Ahora bien, cuando se realizó la correlación parcial entre percepción de enfermedad y calidad de vida, controlando por distrés psicológico, la gran mayoría de las asociaciones se volvieron no significativas (tabla 3). Como se observa, solamente la subescala de Identidad se relaciona significativamente con QLQ-30: Funcionamiento social y la subescala de Comprensión se asocia con QLQ-30: Funcionamiento de Rol.

En la tabla 4 se muestran los resultados del análisis de regresión por pasos para cada escala de funcionamiento de calidad de vida y la escala global. Como puede notarse, la subescala de Comprensión aparece como una variable predictiva en el modelo propuesto para QLQ-30: Funcionamiento de Rol, pero el p-valor del cambio del $\mathrm{R}^{2}$ no es significativo. En cambio la subescala de Identidad, si bien aporta un cambio significativo en el $\mathrm{R}^{2}$ del modelo para QLQ-30: Funcionamiento Social, se observa que dicho $\mathrm{R}^{2}$ es pequeño.

Ahora bien, la inclusión de las variables de Diagnóstico Psicopatológico y Distrés presenta un incremento significativo del $\mathrm{R}^{2}$ en prácticamente todos los modelos, a excepción del de QLQ-30: Funcionamiento de Rol.

\section{Discusión}

El presente estudio tiene como objetivo aportar datos empíricos y mejorar el conocimiento de la relación entre la percepción de enfermedad y la calidad de vida en pacientes con diagnóstico de cáncer de mama, siguiendo y ampliando los resultados obtenidos en otras investigaciones realizadas en pacientes con otro diagnóstico oncológico y otras patologías médicas.

Los resultados del presente estudio pone en discusión la relación directa entre Percepción de Enfermedad y Calidad de Vida, dado que el mayor porcentaje de varianza es explicado por el diagnóstico psicopatológico y el distrés psicológico. Incluso observamos que la variabilidad en la calidad de vida, en una porción importante de los modelos de regresión estudiados, se explicaba más por los niveles de estrés y el diagnóstico psicopatológico que por el estadio de enfermedad y la edad. Consideramos que este hallazgo resalta la importancia de considerar los aspectos psicopatológicos de los pacientes con cáncer, muchas veces subestimados en comparación con otros indicadores más duros, como en el caso del estadio de la enfermedad.

Volviendo particularmente a la relación entre percepción y calidad de vida, los resultados de las investigaciones son controvertidos. En este sentido es importante mencionar una revisión realizada por Quiceno y Vinaccia (29), en donde explicitan los hallazgos contradictorios en torno a la relación mencionada. Si bien señalan que de las investigaciones que evalúan la percepción de enfermedad con la calidad de vida, en particular los estudios longitudinales, se puede evidenciar una estrecha relación entre estas dos variables en estudios longitudinales, mencionan un estudio de Han et al. ${ }^{(30)}$ pacientes diagnosticados de colitis ulcerosa, en el que las percepciones de enfermedad de los pacientes tenían un bajo nivel de predicción de la calidad de vida.

En estudios previos en pacientes con enfermedad cardiovascular y con diagnóstico de cáncer de cabeza y cuello se ha observado que la representación de en- 


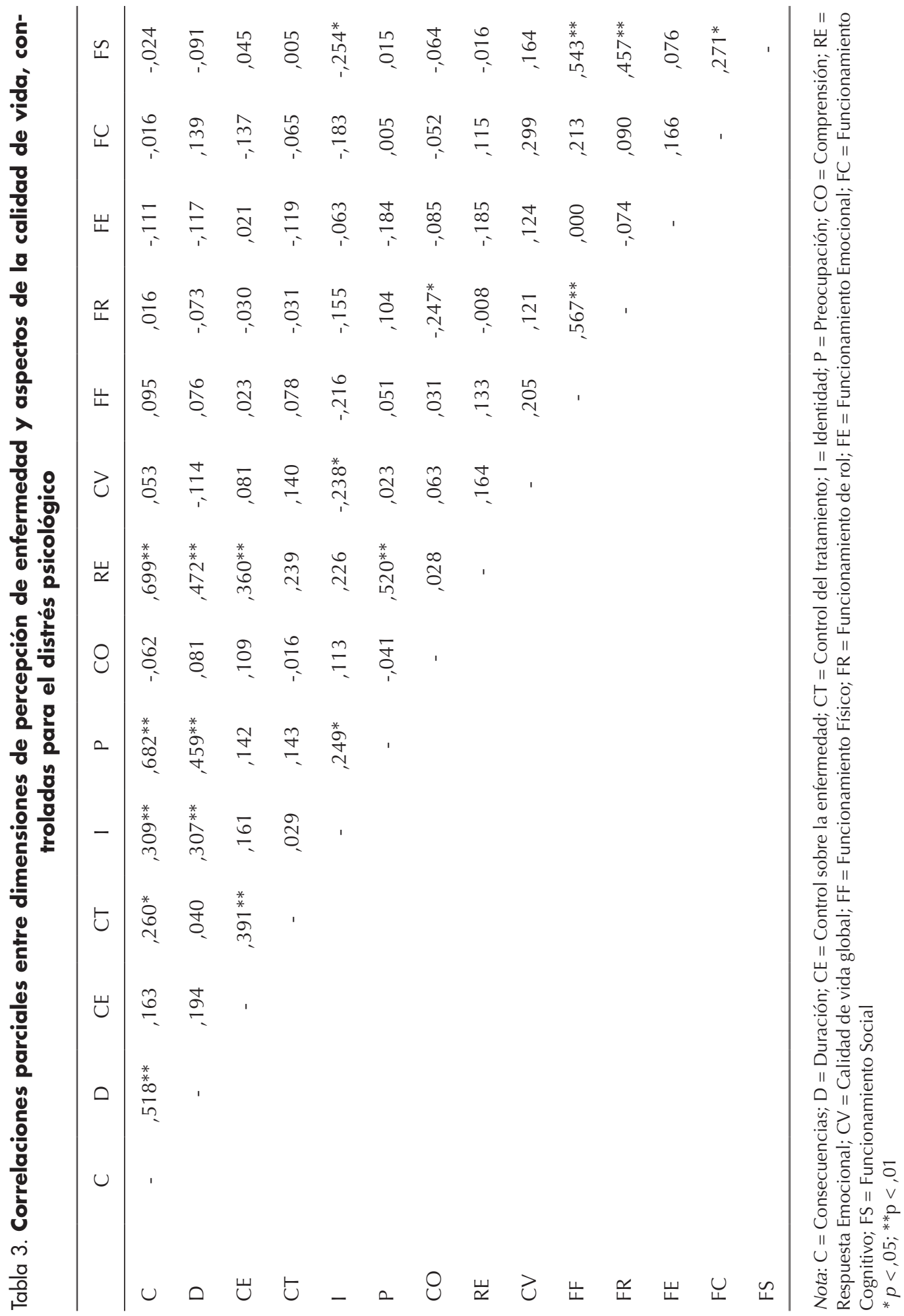


Tabla 4. Análisis de regresión por pasos.

\begin{tabular}{|c|c|c|c|c|}
\hline Paso y Variable & $\mathrm{R}$ & $\mathrm{R}^{2}$ & $\begin{array}{c}\text { Cambio de } \\
\mathrm{R}^{2}\end{array}$ & $\begin{array}{l}\text { F del cambio } \\
\text { p-valor }\end{array}$ \\
\hline \multicolumn{5}{|l|}{ Calidad de Vida Global } \\
\hline $\begin{array}{l}\text { 1. Edad } \\
\text { Estadio de la Enfermedad }\end{array}$ & , 166 & 028 & , 028 &, 380 \\
\hline $\begin{array}{l}\text { 2. Diagnóstico Psicopatológico } \\
\text { HADS }\end{array}$ &, 414 &, 171 &, 144 & ,005 \\
\hline \multicolumn{5}{|l|}{ Funcionamiento Físico } \\
\hline $\begin{array}{l}\text { 1. Edad } \\
\text { Estadio de la Enfermedad }\end{array}$ & ,286 & ,082 & ,82 & 053 \\
\hline $\begin{array}{l}\text { 2. Diagnóstico Psicopatológico } \\
\text { HADS }\end{array}$ &, 442 & ,196 &, 114 & ,012 \\
\hline \multicolumn{5}{|l|}{ Funcionamiento de Rol } \\
\hline $\begin{array}{l}\text { 1. Edad } \\
\text { Estadio de la Enfermedad }\end{array}$ &, 366 &, 134 &, 134 &, 007 \\
\hline $\begin{array}{l}\text { 2. Diagnóstico Psicopatológico } \\
\text { HADS }\end{array}$ &, 410 &, 168 &, 035 & ,255 \\
\hline 3. Comprensión & ,449 & ,202 & 033 &, 101 \\
\hline \multicolumn{5}{|l|}{ Funcionamiento Emocional } \\
\hline $\begin{array}{l}\text { 1. Edad } \\
\text { Estadio de la Enfermedad }\end{array}$ & 130 & 017 &, 017 &, 556 \\
\hline $\begin{array}{l}\text { 2. Diagnóstico Psicopatológico } \\
\text { HADS }\end{array}$ & 0,741 &, 549 &, 532 & $<, 001$ \\
\hline \multicolumn{5}{|l|}{ Funcionamiento Cognitivo } \\
\hline $\begin{array}{l}\text { 1. Edad } \\
\text { Estadio de la Enfermedad }\end{array}$ & 072 & ,005 & ,005 & ,835 \\
\hline $\begin{array}{l}\text { 2. Diagnóstico Psicopatológico } \\
\text { HADS }\end{array}$ &, 594 &, 353 & ,347 & $<, 001$ \\
\hline \multicolumn{5}{|l|}{ Funcionamiento Social } \\
\hline $\begin{array}{l}\text { 1. Edad } \\
\text { Estadio de la Enfermedad }\end{array}$ & ,196 & ,039 & 039 &, 257 \\
\hline $\begin{array}{l}\text { 2. Diagnóstico Psicopatológico } \\
\text { HADS }\end{array}$ &, 370 &, 137 & 098 & 027 \\
\hline 3. Identidad &, 432 & ,186 & ,049 & ,05 \\
\hline
\end{tabular}


fermedad predecía la calidad de vida del paciente, el distrés psicosocial y el retorno al trabajo posterior a las intervenciones médicas ${ }^{(13,31)}$. En nuestro trabajo, sin embargo, el distrés psicosocial sería el principal factor explicativo de los cambios en la calidad de vida de los pacientes, por encima de la edad, el estadio de la enfermedad y las dimensiones de percepción de enfermedad.

Estudios previos que encontraron una alta asociación entre percepción de enfermedad y calidad de vida, como el de Scharloo et al., y el de Buick ${ }^{(9)}$, sugieren que los pacientes con una percepción de enfermedad negativa presentan puntajes inferiores en medidas de calidad de vida antes del tratamiento que podrían aumentar el riesgo de una peor adaptación al tratamiento específico. Sin embargo, dichos estudios no incluyeron medidas de distrés psicológico, razón por la cual no podemos estimar qué hubiese sucedido con dichas asociaciones si esta variable hubiese estado presente.

Consideramos que la relevancia de estudios como el presente radica, en consonancia con estudios previos ${ }^{(14,15)}$, en resaltar el rol que cumplen ciertas variables psicológicas, como el diagnóstico psicopatológico y el distrés psicosocial en la evolución de la enfermedad oncológica y la calidad de vida de los pacientes. En este sentido, diversos estudios ${ }^{(32)}$ sugieren que la depresión aumenta la tasa de morbimortalidad de los pacientes con diagnóstico de cáncer, debido en gran medida al efecto que la depresión tiene sobre el sistema inmunológico.

Como limitación de este estudio podemos mencionar el diseño transversal. Consideramos que es apropiado cotejar los resultados del presente trabajo en un estudio longitudinal en el que puedan incluirse análisis de ecuaciones estructurales que delimiten más claramente la relación entre percepción de enfermedad - distrés psicológico - calidad de vida.

\section{REFERENCIAS BIBLIOGRÁFICAS}

1. Leventhal, H. Diefenbach, M. The active side of illness cognition. In: Skelton JA, Croyle RT, editors. Mental representation in health and illness. New York: SpringerVerlag 1991; p 247-72.

2. Diefenbach MA, Leventhal $\mathrm{H}$. The common-sense model of illness representation: Theoretical and practical considerations. J Social Distress Homeless 1996; 5: 11-38. Doi: 10.1007/BF02090456

3. Bishop GD. Understanding the understanding of illness: lay disease representations. In: Skelton JA, Croyle R.T, editors. Mental representation in health and illness. New York: Springer-Verlag 1991; p. 32-59.

4. Beneit, PJ, Latorre JM. Representación mental de la enfermedad. En: Latorre JM, Beneit PJ, editores. Psicología de la salud. Buenos Aires, Argentina: Lumen 1994; p. 215-24

5. Leventhal H, Leventhal EA, Cameron L. Representations, procedures, and affect in illness self-regulation: A perceptual-cognitive model. In Baum A, Revenson TA, Singer JE, editors. Handbook of health psychology. Mahwah, New Jersey: Lawrence Erlbaum 2001.p. 19-48.

6. Beléndez $M$, Bermejo RM, García D. Estructura factorial de la versión española del Revised Illness Perception Questionnaire en una muestra de hipertensos. Psicothema 2005:17:318-24

7. Petrie KJ. Cameron LD, Ellis CJ, Buick D, Weinman J. Changing illness perceptions after myocardial infarction: an early intervention randomized controlled trial. Psychosom Med 2002; 64: 580-6

8. Hagger MS, Orbell S. A meta-analytic review of the common-sense model of illness representations. Psychol Health 2003; 18:141-84. Doi: 10.1080/088704403100081321

9. Buick DL. Illness representations and breast cancer: Coping with radiation and 
chemotherapy. In: Petrie KJ, Weinman J, editors. Perceptions of health and illness. Amsterdam: Harwood Academic 1997.p. 379-410

10. Marteau TM, Rana S, Kubba A. Smoking and cervical cancer: A qualitative study of the explanatory models of smokers with cervical abnormalities. Psychol Health Med 2002; 7:107-9. Doi: 10.1080/13548500120101504

11. Silva SM, Moreira HC, Canavarro MC. Examining the links between perceived impact of breast cancer and psychosocial adjustment: The buffering role of posttraumatic growth. Psychooncology 2012; 21(4):409-18. Doi: 10.1002/pon.1913

12. Browning KK, Wewers ME, Ferketich AK, Otterson GA, Reynolds NR. The SelfRegulation Model of Illness applied to smoking behavior in lung cancer. Cancer Nurs 2009;32(4):E15-E2. Doi: 10.1097/ NCC.0b013e3181a0238f

13. Scharloo M, Baatenburg de Jong RT, Langeveld TPM, van Velzen-Verkaik E. Doorn-op den Akker MM, Kaptein AA. Quality of life and illness perceptions in patients with recently diagnosed head and neck cancer. Head Neck 2005; 27(10): 857-63. Doi: 10.1002/hed.20251

14. Ganz PA. Psychological and social aspects of breast cancer. Oncology (Williston Park). 2008; 22 (6): 642-6, 650

15. Wang L, van Belle G, JKukull WB, Larson EB. Predictors of functional change: A longitudinal study of nondemented people aged 65 and older. J Am Geriatr Soc 2002; 50 (9): 1525-34. Doi: 10.1046/j.15325415.2002.50408.x

16. Martins Silva, S., Cruz Moreira H, Canavarro MC. Examining the links between perceived impact of breast cancer and psychosocial adjustment: the buffering role of posttraumatic growth. Psychooncology 2012; 21: 409-8. Doi: 10.1002/pon.1913

17. Montero, I. y León, O. G. A guide for naming research studies in Psychology. Int J Clin Health Psychol 2007;7:847-62
18. Broadbent E, Petrie KJ, Main J, Weinman J. The Brief Illness Perception Questionnaire. J Psychosom Res 2006; 60: 631-7. Doi: 10.1016/j.jpsychores.2005.10.020

19. Pacheco-Huergo V, Viladrich C, Pujol-Ribera $E$, Cabezas-Peña $C$, Núñez $M$, RouraOlmeda $\mathrm{P}$, et al. en representación del Grupo IPQ-R. Perception in chronic illnesses. Linguistic validation of the revised Illness Perception Questionnaire and the Brief Illness Perception Questionnaire for a Spanish population. Aten Primaria 2012; 44 (5): 280-7

20. Pacheco-Huergo, V. Medida de percepción en enfermedades crónicas: Adaptación cultural de los cuestionarios Illness Perception Questionnaire Revised (IPQ-R) y Brief Illness Perception Questionnaire (BIPQ) para la población española. Tesis Doctoral. Universidad Autónoma de Barcelona. 2012

21. Sprangers MA, Cull A, Bjordal K, Groenvold M, Aaronson NK. The European Organization for Research and Treatment of Cancer. Approach to quality of life assessment: guidelines for developing questionnaire modules. EORTC Study Group on Quality of Life. Qual Life Res 1993;2(4): 287-95

22. Arrarás J I, Illaramendi JJ, Valerdi JJ. El cuestionario de calidad de vida para cáncer de la EORTC, QLQC-30. Estudio estadístico de validación con una muestra española. Rev Psicol Salud 1995; 7: 13-31.

23. Zigmond AS, Snaith RP. The Hospital Anxiety and Depression Scale. Acta Psychiatr Scand 1983; 67: 361-70. Doi:10.1111/j.1600-0447.1983.tb09716.x

24. Herrmann C. International experiences with the Hospital Anxiety and Depression Scale - a review of validation data and clinical results. J Psychosom Res 1997; 42 (1): 17-41. Doi: 10.1016/S00223999(96)00216-4

25. Bjelland I, Dahl A.A, Haug TT, Neckelmann D. The validity of the Hospital Anxiety and Depression Scale; an updated review. J 
Psychosom Res 2002; 52 (2): 69-77. Doi: 10.1016/S0022-3999(01)00296-3

26. Vodermaier A, Millman RD. Accuracy of the Hospital Anxiety and Depression Scale as a screening tool in cancer patients: A systematic review and meta-analysis. Support Care Cancer 2011; 19 (12):1899908. Doi:10.1007/s00520-011-1251-4

27. Gercovich D, Torrente F, López P, Bortolato D, Margiolakis P, Morgenfeld M. Evaluación de propiedades psicométricas de la escala de ansiedad y depresión hospitalaria en pacientes oncológicos de Buenos Aires. Acta Psiquiatr Psicol Am Lat 2009; 55 (2): 84-91

28. American Psychiatric Association. Diagnostic and Statistical Manual of Mental Disorders, 4th edition - text revision (DSM-IV-TR). Washington, DC: American Psychiatric Association, 2000.

29. Quiceno JM, Vinaccia S. Percepción de
Enfermedad: Una aproximación a partir del Illness Perception Questionnaire. Psicología desde el Caribe 2010; 25: 56-83

30. Han SW, McColl E, Barton JR, James P, Steen IN, Welfare MR. Predictors of quality of life in ulcerative colitis: The importance of symptoms and illness representations. Inflamm Bowel Dis 2005; 11 (1):2434. Doi: 10.1097/00054725-20050100000004

31. Hermele S, Olivo EL, Namerow P, Oz MC. Illness representations and psychological distress in patients undergoing coronary artery bypass graft surgery. Psycholo Health Med 2007; 12: 580-91. Doi: 10.1080/13548500601162705

32. Croyle RT. Depression as a risk factor for cancer: Renewing a debate on the psychobiology of disease. JNCI J Natl Cancer Inst 1998; 90 (24): 1856-7. Doi: 10.1093/ jnci/90.24.1856 